\title{
ONDE SUPERFICIALI ATTRAVERSO IL BACINO DELL'ATLANTICO (*)
}

\author{
P. Calor - L. Marceill
}

1. - Doblbiamo una breve replica ad alcune obiezioni $\left({ }^{1}\right)$ :

a) Le onde da noi indicate con $L_{Q}$ nei lavori precedenti (2-3) non possono, in nessun caso, essere confuse con $S S, S S S$, sia per i tempi di tragitto, sia per i relativi periodi.

b) Per le ragioni esposte nella seconda nota sull'argomento ( ${ }^{3}$ ), non $\dot{e}$ consentito attribuire le stesse caratteristiche elastiche agli opposti retroterra dell'Atlantico.

Ciò nonostante, come si ̀̀ pure osservato nel lavoro ora citato, a conferma della grande velocità delle onde $L_{Q}$, anche nel bacino orientale dell'Atlantico, oltre alla stazione di Lisbona, c'è la testimonianza ben più incontestalıile di Kew, dove le onde $L_{Q}$ appaiono netlissime e mostrano una velocità ancora più grande di quella registrata ad Ottawa, di $4,3 \mathrm{~km} / \mathrm{sec}$ almeno.

Ma c’è di più. Per quanto concerne le registrazioni di Tortosa e Barcellona, come del resto abliamo già osservato $\left({ }^{3}\right)$, per un eccesso di prudenza ablbiamo, in un primo tempo, posto l'inizio delle $I_{Q}$ dove ci parve più sicuro. Uno sguardo a dette registrazioni prova però che l'inizio delle onde stesse poteva essere anticipato di oltre un minuto. Per Barcellona, p. es., ponendo l'inizio delle ' $L_{Q}$ come da fig. 1 , la velocità di queste onde risulta di $4,1 \mathrm{~km} / \mathrm{sec}$, dell'ordine cioè di quella osservata nell'opposto retroterra dell'Atlantico, a Columbia e a Ottawa, non ostante il periodo osservato a Barcellona sia soltanto di 18 sec circa. Analogo il comportamento a Tortosa, dove le onde superficiali possono essere anticipate di $62 \mathrm{sec}$, il che porta la loro velocità a $4,2 \mathrm{~km} / \mathrm{sec}$ ca. Le insufficienti registrazioni di Jersey (dove funzionano strumenti di poca sensibilita) sono neutralizzate dalle nitide registrazioni di $\mathrm{Kew}$, che si trova presso a poco sullo stesso azimut e a maggior distanza, dove come si $\dot{e}$ detto $\left(^{3}\right)$ le onde $L_{\mathrm{Q}}$ mostrano una elevata velocità di propagazione, maggiore di quella osservata ad Ottawa. Il fatto che a Tortosa e a Barcellona precedano le onde di minor

(*) Comunicazione presentata al Convegno dell'Associazione Geofisica Italiana, tenuto a Roma il 10.11 giugno 1952. 


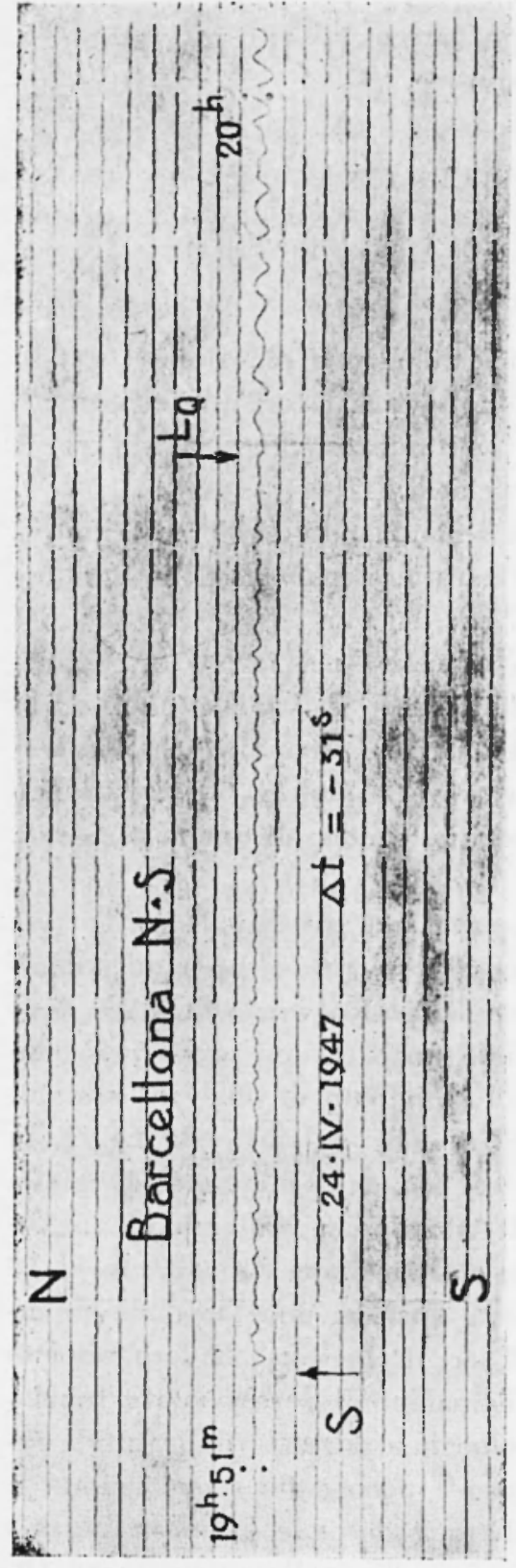

periodo, può spiegarsi con la teoria dei fronti-guida: l'onda proveniente dall'Atlantico pro. segue il suo tragitto sotto lo zoccolo continentale, dove intanto nasce l'onda a periodo maggiore, ma inizialmente di minore velocità, attesa la elasticità ridotta dello strato continentale.

c) A proposito della presunta saldatura della cinta sismica mediterranea con le Azzorre, non si vede come, se essa esiste, possa testimoniare della cquivalenza geologica di estese regioni quali l'Europa, l'Africa e '’Atlantico orientale.

La sismicità nei pressi delle Azzorre è del tutto diversa da quella della penisola Iberica. Inoltre, la notevole densità degli epicentri che si osserva lungo tutta la dorsale centrale atlantica, paragonata a quella debolissima della fossa fra le Azzorre e la penisola Iberica, costituisce una prova chiaris. sima del difetto di saldatura fra le due zone sismiche del Mediterraneo e della dorsale atlantica.

d) Non solamente le due zone dell'Atlantico hanno una hatimetria comparabile, ma quasi uguale ̀̀ anche la loro sismicità.

e) La teoria di Wegener è difficilmente sostenibile. Nel caso specifico, se la separazione delle Americhe si fosse verificata lungo la dorsale atlantica, le enormi fratture così determinate, avrebbero dovuto 


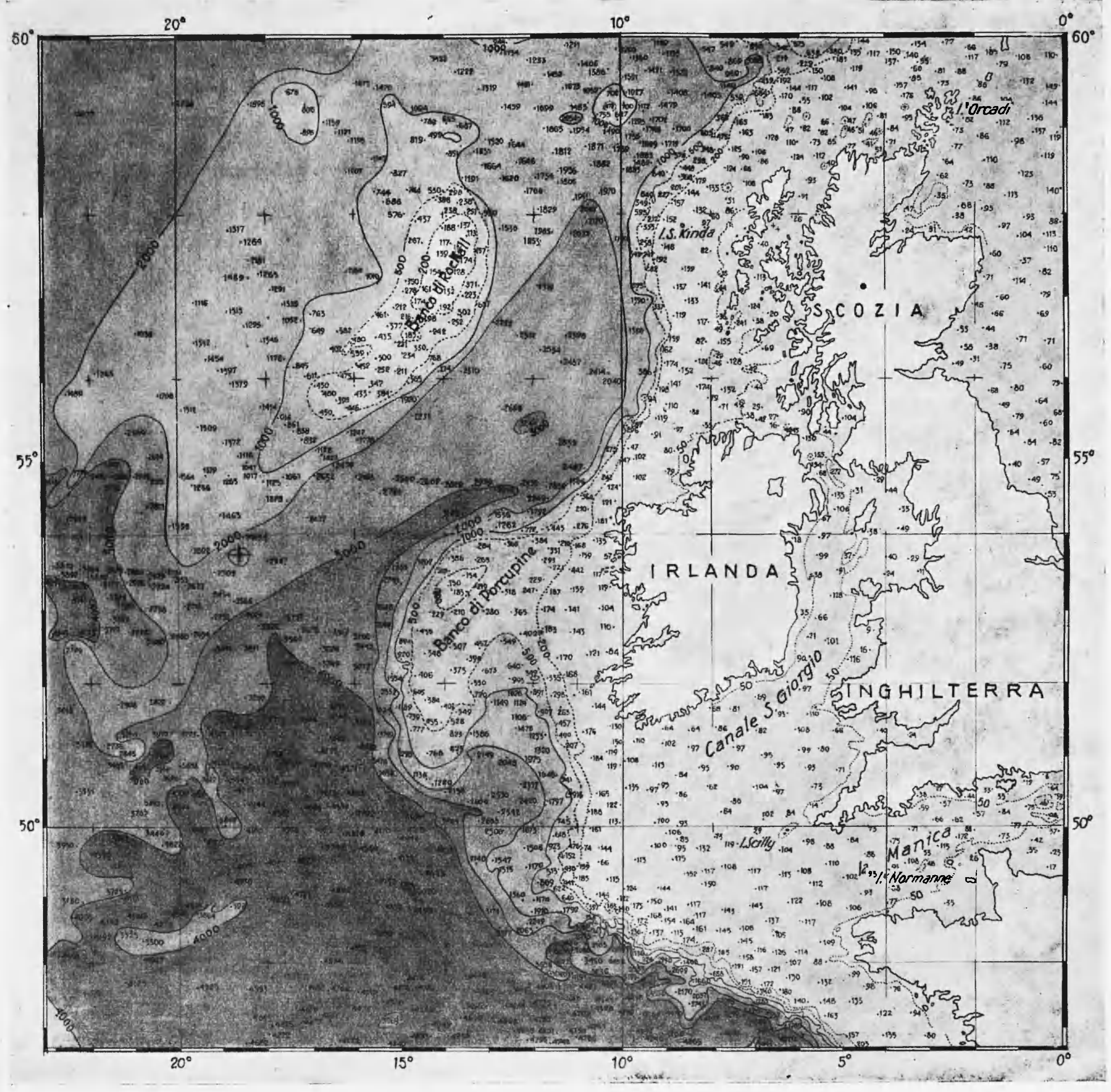

Fig. 2 
produrre sul lato occidentale della dorsale una scarpata, con pendio più o meno accentuato; mentre a oriente il fondo dell'Oceano dovrebbe risultare alquanto più sollevato.

La dorsale, al contrario, giustifica in pieno il suo nome ed ha tutte le caratteristiche d'una montagna sommersa.

La morfologia non testimonia dunque né per la rottura, né per l'allontanamento.

f) Se non proprio un sottile strato continuo, almeno delle isole sialiche possono ben esistere sul fondo dell'Atlantico. Un tale strato, però, se esiste, dev'essere estremamente sottile: da uno spessore di $40 \mathrm{~km} \mathrm{ca}$. in corrispondenza del sistema alpino, esso diviene di 20 km ca. per il Württemberg, di $16 \mathrm{~km}$ ca. per la Renania, per ridursi a meno di $10 \mathrm{~km}$ per le isole britanniche.

A questo riguardo osserviamo che $\mathrm{i}$ risultati delle prospezioni sismiche per rifrazione, ottenuti da Maurice N. Hill $\left({ }^{8}\right)$ intorno al punto $53^{\circ} 50^{\prime} \mathrm{N}, 18^{\circ} 40^{\prime} \mathrm{W}$, si riferiscono ad una zona che non ha nulla a che vedere con la dorsale atlantica; la zona in questione, infatti, si trova ai margini meridionali del Banco di Rockall e presso il Banco di Porcupine, vale a dire in una posizione prossima allo zoccolo continentale (fir. 2). Ebbene, in questa zona, secondo le valutazioni di Hill, ad uno strato di sedimenti sciolti di uno spessore variante dai 1900 ai $3000 \mathrm{~m}$, segue uno strato cristallino caratterizzato da una velociti di $5,00 \mathrm{~km}$ 'sec per le onde longitudinali, avente uno spessore dell'ordine di $3,4-2,7 \mathrm{~km}$. Ai margini dello zoccolo, lo strato continentale si è quindi notevolmente assottigliato. $\dot{E}$ pertanto logico ritenere che, più ad occidente e a sud, dove la profondità dell'Oceano è prossima ai $5000 \mathrm{~m}$, tale strato si riduca ulteriormente e finisca con lo scomparire del tutto.

g) La scoperta di materiali sialici in qualche punto sul fondo dell'Atlantico orientale, non è affatto argomento decisivo a favore dell'ipotesi sulla differenza geologica fra Atlantico orientale e Atlantico occidentale. Il geologo portoghese C. Teixeira fa sull'argomento una analisi sottile $\left({ }^{{ }^{4}}\right)$ e prova la possibilità della provenienza continentale di tali materiali. Riguardo all'ipotesi citata, egli è esplicito ed afferma che essa è insostenibile.

Lo stesso Maurice Ewing, alcuni lavori del quale erano stati invocati a favore dell'ipotesi sulla differenza geologica delle zone atlantiche ad est $e$ ad ovest della dorsale mediana, in un recente studio, compiuto con Frank Press ("), sulla struttura della crosta alla base 

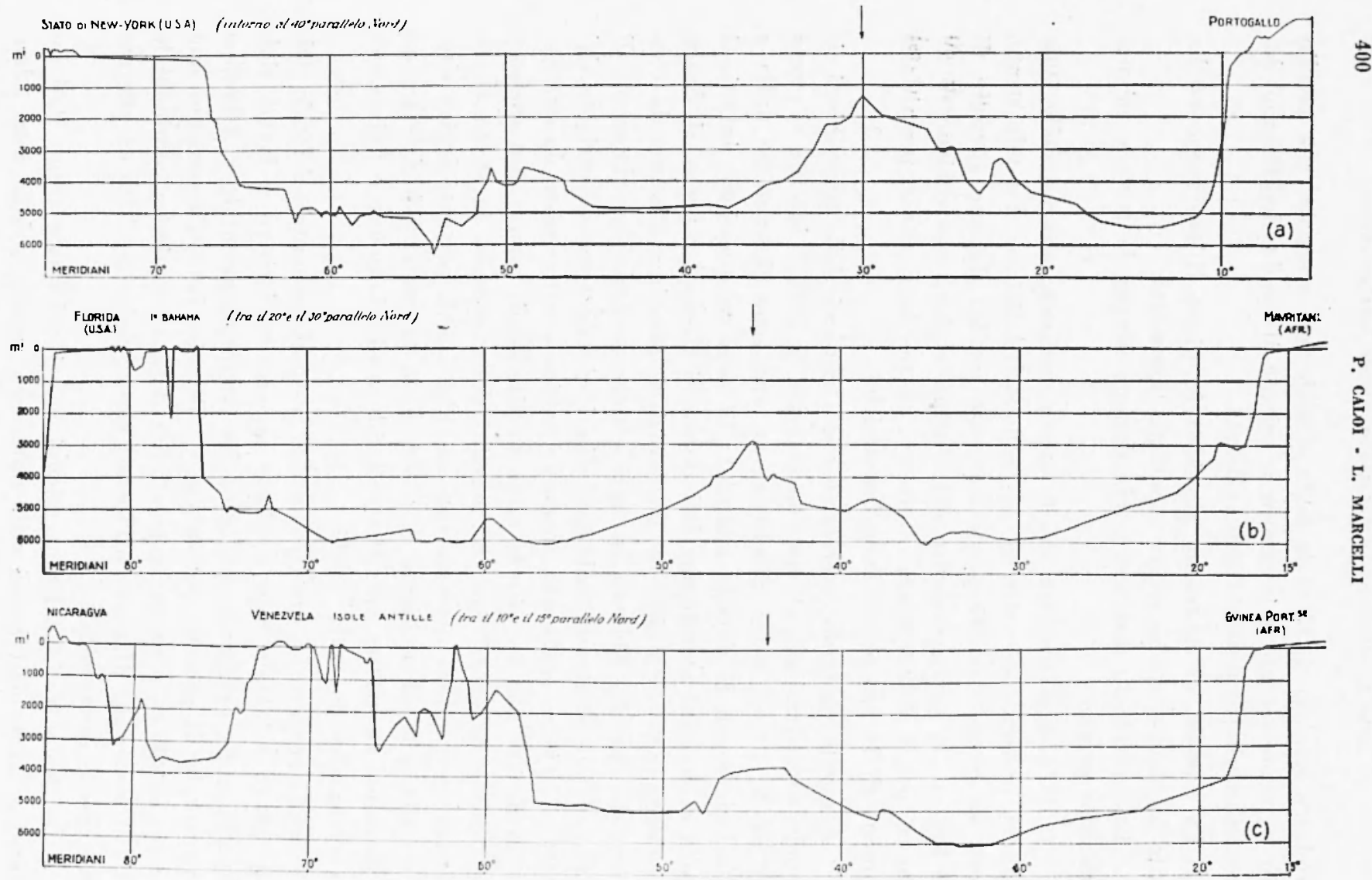

Fig. 3 
ONDE SUPERFICIALI ATtRAVERSo IL BaCINo deLL'AtLANTICo
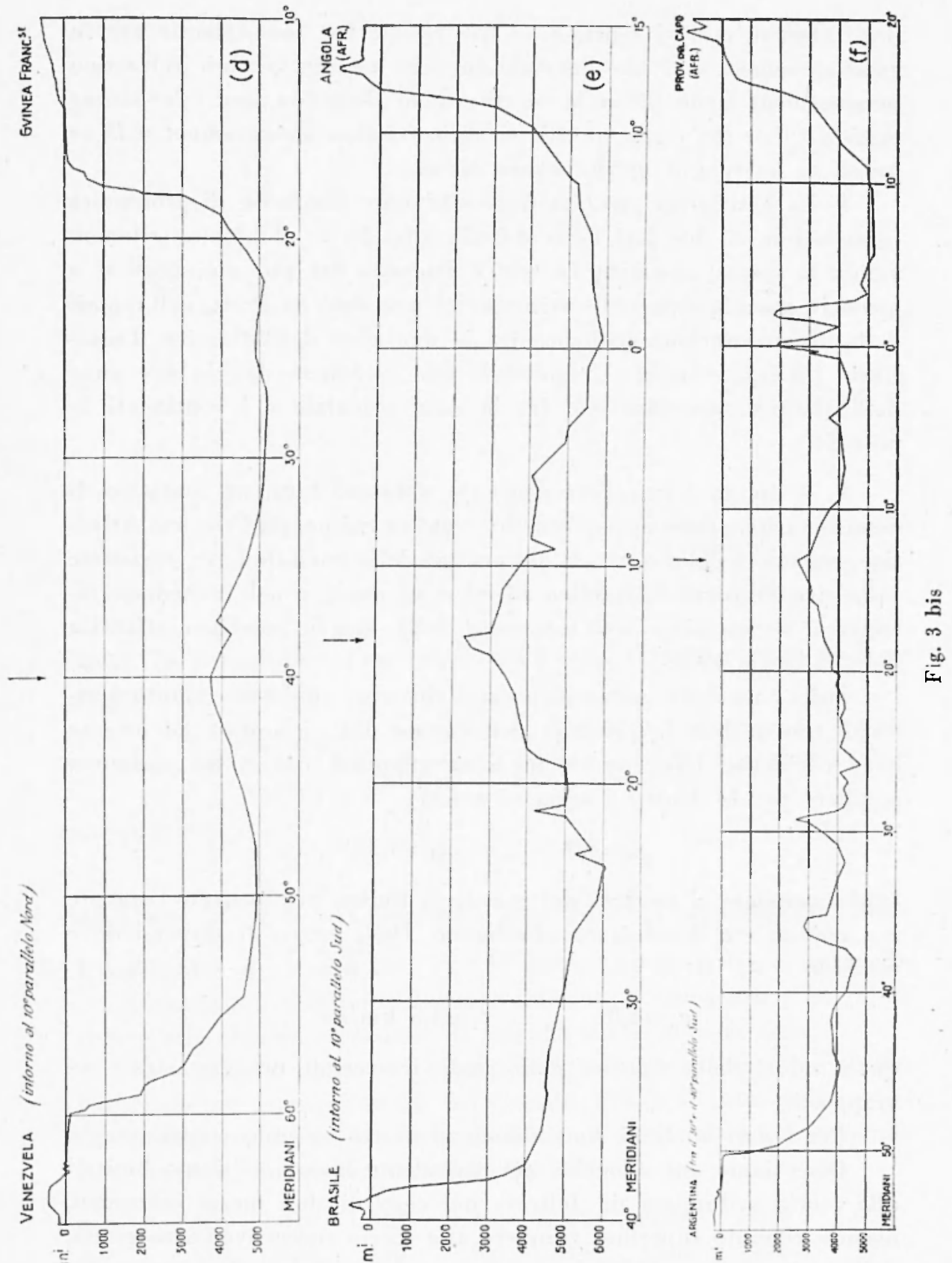
degli Oceani, cosi si esprime: "The results for the Atlantic are in good agreement with the data obtained in a recent seismic refraction measurement made 120 miles northwest of Bermuda, and offer strong evidence that the result of this single refraction measurement will be found to be typical of the entire ocean".

h) La batimetria presenta fosse e pianure sommerse di profondità comparabile ai due lati della dorsale (fig. 3). Se dobbiamo ritenere valida la teoria isostatica (e tale è ritenuta dai più noti geodeti e geologi), specialmente nello sviluppo ad essa dato da Pratt, nell'ipotesi della differenziazione geologica fra le due zone dell'Atlantico, l'equilibrio isostatico sarebbe impossibile non solamente fra le due zone dell'Atlantico, ma, ben più, fra la zona orientale e i continenti limitrofi.

2. - In un lavoro precedente $\left({ }^{3}\right)$, abbiamo fatto un tentativo di determinazione dello spessore dello strato su cui poggia l'Oceano Atlantico, valendoci delle curve di dispersione delle onde di Love, registrate sulle rive limitanti l'Atlantico ad est e ad ovest, o nell'immediato retroterra, in occasione del terremoto della dorsale mediana atlantica del 24 Aprile 1947.

Sulla base delle nostre precedenti ricerche, abbiamo ritenuto pressoché trascurabile la presenza dello strato del "granito" ad oriente e ad occidente della dorsale mediana atlantica. Ciò ci ha portato a scegliere per le densità $i$ seguenti valori:

$$
\varrho_{\mathrm{o}}=3,0 \quad ; \quad \varrho_{\mathrm{i}}=3,32 \quad \text {, }
$$

conformemente ai risultati dei calcoli di Bullen per lo strato seguente il " granito » e il substrato ultrabasico. Dalle curve di dispersione è risultato

$$
c_{\mathrm{o}}=4,0 \quad, \quad c_{\mathrm{i}}=4,5 \mathrm{~km} / \mathrm{sec} \text {, }
$$

quali valori della velocità delle onde trasversali nei due strati sovrapposti.

Per i dati analitici rimandiamo al nostro secondo contributo $\left({ }^{3}\right)$.

Osserviamo qui solo che, nel precedente lavoro, ci siamo limitati alla teoria sviluppata da Jeffreys nel caso di due mezzi omogenei, avendo ritenuto superfluo ricorrere alla teoria successivamente estesa, dallo stesso Jeffreys $\left(^{6-7}\right)$, al caso di un mezzo (il secondo) eterogeneo.

Allo scopo di dare carattere di completezza alla nostra ricerca, abbiamo fatto ricorso anche a questa estensione della teoria di Jef- 


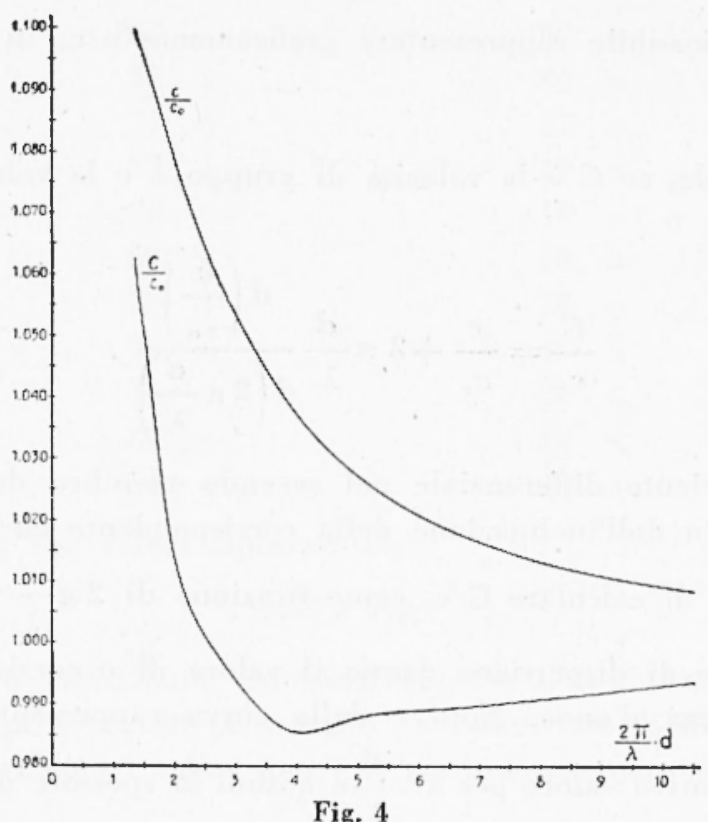

freys. In questo caso, l'equazione della velocità per le onde di Love assume la forma $\left({ }^{6}\right)$.

$\operatorname{tang} k . a_{\mathrm{o}} d=\frac{\ddot{H}_{\mathrm{i}}}{\mu_{\mathrm{o}}} \frac{a_{\mathrm{i}}}{a_{\mathrm{o}}}\left\{1+\frac{1}{2 k L a_{\mathrm{i}}}\left(1+\frac{1}{a_{\mathrm{i}}^{2}}\right)-\frac{1}{8 k^{2} L^{2} a_{\mathrm{i}}{ }^{(}}\left(1-a_{\mathrm{i}}{ }^{2}\right)\left(5+a_{\mathrm{i}}{ }^{2}\right)+\right.$ $\left.+0(k L)^{-3}\right\}$,

dove $k=\frac{2 \pi}{\lambda}, L$ è una lunghezza costante e gli altri simboli hanno il significato espresso dalle [1], [3] del nostro precedente lavoro $\left({ }^{3}\right)$. Quando $k L$ è infinito il mezzo inferiore è omogeneo e la [1] si riduce alla classica equazione di velocità delle onde di Love.

La soluzione della [1] è stata proposta al benemerito Istituto Nazionale per le applicazioni del Calcolo. Fra le infinite radici positive e negative della [1], corrispondenti ad ogni valore del rapporto c/co fanno al nostro caso quelle qui riportate:

$\begin{array}{lrrrrrrrc}\mathrm{c} / \mathrm{c}_{\mathrm{o}} & 1.005 & 1.010 & 1.0125 & 1.020 & 1.025 & 1.030 & 1.040 & 1,050 \\ \boldsymbol{k} & .828 & .560 & .490 & .366 & .314 & .276 & .224 & .187 \\ \mathrm{c} / \mathrm{c}_{\mathrm{o}} & 1.060 & 1.070 & 1.080 & 1.085 & 1.090 & 1.095 & 1.098 & 1.100 \\ k & .158 & .135 & .116 & .107 & .0979 & .0891 & .0834 & .0788 .\end{array}$


É così possibile rappresentare graficamente $c / c_{o}$ in funzione di $\frac{2 \pi}{\lambda} d$.

D'altronde, se $C$ è la velocità di gruppo e $c$ la velocità di fase, si ha $\left({ }^{3}\right)$ :

$$
\frac{C}{c_{\mathrm{o}}}=\frac{c}{c_{\mathrm{o}}}+2 \pi \frac{d}{\lambda} \frac{d\left(\frac{c}{c_{\mathrm{o}}}\right)}{d\left(2 \pi-\frac{d}{\lambda}\right)} .
$$

Il coefficiente differenziale nel secondo membro della [2] può essere dedotto dall'inclinazione della corrispondente curva per $c / c_{0}$; ciò permette di calcolare $\mathrm{C} / \mathrm{c}_{\mathrm{o}}$ come funzione di $2 \pi \frac{d}{\lambda}$ (fig. 4$)\left({ }^{*}\right)$.

Le curve di dispersione danno il valore di $c$ corrispondente ad ogni lunghezza d'onda. Noto $c$ dalla curva rappresentante $\mathrm{C} / \mathrm{c}_{\mathrm{o}}$ si deduce il relativo valore per $2 \pi \frac{d}{\lambda}$ e quindi lo spessore $d$ dello strato.

Si sa che, in questo genere di misure, non è possibile raggiungere valori molto approssimati, specie per ciò che si riferisce alla lunghezza d'onda. Ecco, ad ogni modo, i valori ottenuti per $d$ :

\begin{tabular}{|c|c|c|c|}
\hline \multirow{5}{*}{ Harvard } & $\lambda$ & $d$ & \\
\hline & $76 \mathrm{~km}$ & 17 & km \\
\hline & $68 》$ & 17 & ” \\
\hline & 58 & 16 & $\eta$ \\
\hline & 58 & 18 & 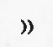 \\
\hline \multirow[t]{4}{*}{ Scoresbv Sund } & 66 & 17 & $\eta$ \\
\hline & 61 & 18 & $\eta$ \\
\hline & 52 & 17 & 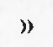 \\
\hline & 48 & 17 & $\eta$ \\
\hline \multirow[t]{4}{*}{ Lisbona } & 76 & 16 & $n$ \\
\hline & 63 & 16 & $\eta$ \\
\hline & 70 & 19 & $\eta$ \\
\hline & 65 & 20 & ) \\
\hline \multirow[t]{2}{*}{ Halifax } & 92 & 20 & 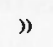 \\
\hline & $101 "$ & 24 & $\eta$ \\
\hline
\end{tabular}

(*) Nel lavoro precedente, nel dare forma definitiva al grafico riportato in fig. 3 , si è fatto un errore nella valutazione delle ordinate (errore senza conseguenze ai fini del calcolo). La fig. 3 del suddetto lavoro, va pertanto sostituita con la fig. 5 . 


\begin{tabular}{|c|c|c|c|}
\hline \multirow[t]{2}{*}{ San Juan } & 108 & $\eta$ & 28 \\
\hline & 82 & " & 30 \\
\hline \multirow[t]{4}{*}{ Kew Obs. } & 77 & " & 17 \\
\hline & 80 & $\eta$ & 19 \\
\hline & 70 & 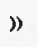 & 19 \\
\hline & 69 & ) & 20 \\
\hline \multirow[t]{5}{*}{ Ottrives } & 85 & $"$ & 19 \\
\hline & 75 & $"$ & 19 \\
\hline & 70 & ) & 20 \\
\hline & 57 & $"$ & 18 \\
\hline & 56 & ) & 20 ) \\
\hline
\end{tabular}

La media dei valori riportati è

$$
d=19 \mathrm{~km} \text {. }
$$

Nel lavoro precedente, avevamo ottenuto $d=17 \mathrm{~km}$.

I valori qui riportati presentano però carattere di maggiore uniformita. Infatti, solo i valori dati da San Juan scartano sensibilmente dalla media.

Non si è tenuto conto dei valori tratti dalle registrazioni di Bermuda, che, anche questa volta, sono stati estremamente piccoli (e cioè: per $\lambda=40 \mathrm{~km}, d=9 \mathrm{~km} ; \lambda=48, d=13 ; \lambda=39, d=11$; $\lambda=34, d=11 ; \lambda=35, d=12$ ).

Restano cosi confermati l'ordine di grandezza ottenuto per lo spessore dello strato sopportante l'Atlantico e la stretta analogia che esso presenta con l'analogo strato sopportante il Pacifico. Un'ulteriore conferma della validità di questo risultato, da noi ottenuto nel 1949 , è data dal già citato recente lavoro di Ewing e Press $\left({ }^{5}\right)$. I due studiosi americani concludono, trattando l'argomento per una via diversa

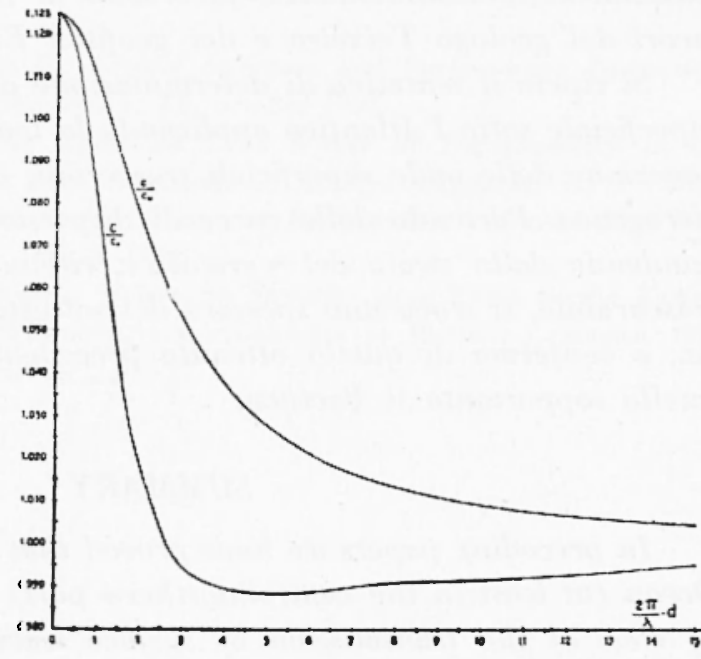

Fig. 5 
dalla nostra: "No significant difference in the nature of the suboceanic basement of the Atlantic and Pacific has been found, since the velocity of shear waves was calculated to $4,45 \mathrm{~km} / \mathrm{sec}$ for both oceans. Previously reported differences in Atlantic and Pacific velocity for Rayleigh waves of some selected period are now believed to he due primarily to differences in the depth of water plus sediment in the two oceans".

Conclusioni identiche a quelle da noi precedentemnte ottenute, per quanto riguarda il fondo dei due Oceani Atlantico e Pacifico. Per ciò che si riferisce alla spiegazione delle precedenti contrastanti conclusioni tratte sulla velocità di propagazione delle onde di Rayleigh, abbiamo avuto modo di provare $\left({ }^{3}\right)$ che esse vanno principalmente attribuite al fatto che $i$ casi in precedenza esaminati si riferivano ad onde di Rayleigh che solo in parte interessavano l'Atlantico, nel loro tragitto.

Roma - Istituto Nazionale di Geofisica - Giugno 1952.

\section{RIASSUNTO}

In precedenti lavori abbiamo provato che il bacino dell'Atlantico (almeno nella sua parte centro-settentrionale) si comporta, dal punto di vista della trasmissione delle onde superficiali, in maniera del tutto analoga al bacino del Pacifico.

Si portano nuovi argomenti a favore di detta conclusione e si riassumono le testimonianze, favorevoli ai nostri risultati, tratte da lavori del geologo Teixeira e dei geofisici Ewing e Press.

Si ripete il tentativo di determinazione dello spessore dello strato superficiale sotto l'Atlantico applicando la teoria di Jeffreys sulla propagazione delle onde superficiali trasversali, che considera il $2^{n}$ strato eterogeneo. Partendo dalle curve di dispersione delle onde $\mathrm{L}_{\mathrm{q}}$ e prescindendo dallo strato del "granito", ritenuto mancante o di entità trascurabile, si trova uno spessore del sottostante strato pari a $19 \mathrm{~km}$ ca., a conferma di quello ottenuto precedentemente e dell'ordine di quello sopportante il Pacifico.

\section{SUMMARY}

In preceding papers we have proved that the basin of the Atlantic Ocean (at least in the central-northern part) behaves, from the point of view of the transmission of surface waves, in a way completely similar to the Pacific basin. 
We state here new arguments in favor of this conclusion, and include a summary of the confirming results of the geologist Teixeira and the geophysicists Ewing and Press.

A new attempt has been made to determine the depth of the first layer under the Atlantic. The theory of Jeffreys, that considers the second layer to be heterogeneous, is applied to the propagation of surface transversal waves. Starting from the curve of dispersion of the wave $\mathrm{I}_{\mathrm{I}}$ and leaving out of consideration the layer of "granite. considered to be missing or of negligible size, one finds a thickness of the underlying layer equal to about $19 \mathrm{~km}$ in confirmation with the value obtained previously, and of the same order as that supporting the Pacific.

\section{BIBLIOGRAFIA}

(1) Rothé J. P.: La structure de l'Atlantique. "Annali di Geofisica », IV, 1 (1951).

(2) Caloi P., Marcelli L., Pannocchia G.: Sulla velocità di propagazione delle onde superficiali in corrispondenza dell'Atlantico. "Annali di Geofisica 》, II, 3 (1949).

(3) Caloi P., Marcell I L. Pannocchia G.: Ancota sulla velocità di propagazione delle onde superficiali per tragitti subatlantici... "Annali di Geofisica ", III, 2 (1950).

(4) Teixeira C.: A propos d'une hypothese sur la structure de l'Océan Atlantique. "Boletin Museum e Laboratório Mineralögico e Geológico », Universidade Lisboa, n. 18 (1950).

(5) Ewing M. and Press F.: Crustal Structure and Surface-uave Dispersion. "Bull. Seismolog. Society of America ", XL, october 1950.

(6) Jefrneys HaroLd: The Effect on Love Waves of Heterogeneity in the Lower Layer. "Monthl. Not. Roy. Astr. Soc.", Geophys. Supplem, II, 2 (1928).

(7) Jefrreys Harold: The Surface Waves of Earthquakes. "Monthl. Not. Roy. Astr. Soc. ", Geophys. Suppl., III, (1935).

(8) Hill Maunice N.: Seismic Refraction Shooting at a Point in the Eastern Atlantic. "Ass. d'Océanogr. Physique, Gen. Assembly at Brussels ", August 1951. Reports and Abstr. of Communic., p. 55. 